\title{
Rendre la conservation de l'agrobiodiversité mexicaine gouvernable ou le difficile transcodage des savoirs scientifiques
}

Making mexican agrobiodiversity conservation governable or the difficult transcoding process of scientific knowledge

Gobernanza de la conservación de la agrobiodiversidad mexicana: la difícil transcodificación de los saberes científicos

Jean Foyer et Marianna Fenzi

\section{OpenEdition}

\section{Journals}

Édition électronique

URL : https://journals.openedition.org/ethnoecologie/7405

DOI : 10.4000/ethnoecologie.7405

ISSN : 2267-2419

Éditeur

Laboratoire Éco-anthropologie

\section{Référence électronique}

Jean Foyer et Marianna Fenzi, « Rendre la conservation de l'agrobiodiversité mexicaine gouvernable ou le difficile transcodage des savoirs scientifiques », Revue d'ethnoécologie [En ligne], Supplément 2 I 2021, mis en ligne le 25 novembre 2021, consulté le 20 décembre 2021. URL : http:// journals.openedition.org/ethnoecologie/7405; DOI : https://doi.org/10.4000/ethnoecologie.7405

Ce document a été généré automatiquement le 20 décembre 2021.

Revue d'ethnoécologie est mis à disposition selon les termes de la licence Creative Commons Attribution - Pas d'Utilisation Commerciale - Pas de Modification 4.0 International. 


\title{
Rendre la conservation de l'agrobiodiversité mexicaine gouvernable ou le difficile transcodage des savoirs scientifiques
}

\author{
Making mexican agrobiodiversity conservation governable or the difficult \\ transcoding process of scientific knowledge \\ Gobernanza de la conservación de la agrobiodiversidad mexicana: la difícil \\ transcodificación de los saberes científicos
}

Jean Foyer et Marianna Fenzi

\section{Introduction ${ }^{1}$}

1 L'opposition au maïs transgénique et la défense des maïs locaux constituent un axe de mobilisation d'acteurs variés réunissant organisations environnementales, paysannes, scientifiques, indigènes ou encore de consommateurs (Marielle \& Peralta 2007, AlvarezBuylla \& Pineyro Nelson 2013, Foyer 2015). Ces coalitions d'acteurs ont su créer un rapport de force pour que les autorités mexicaines prennent en considération une partie de leurs revendications. Ainsi, la Loi de Biosécurité Mexicaine de 2005 reconnaît dans son article 86 la nécessité pour les ministères de l'agriculture (SAGARPA ${ }^{2}$ ) et de l'environnement (SEMARNAT) d'établir un inventaire exhaustif et une cartographie précise de la diversité des maïs mexicains, mais également de prendre des mesures pour leur conservation. La SEMARNAT lance ainsi en 2008 le Programa de Conservación de Maíz Criollo (PROMAC ${ }^{3}$ ) à travers l'une de ces dépendances, la Comisión Nacional de Areas Naturales Protegidas (CONANP). Le PROMAC représente l'initiative institutionnelle de conservation du maïs in situ la plus importante jamais réalisée au Mexique. L'objectif 
affiché de ce programme est de «promouvoir la conservation et la récupération des races et variétés de maïs natif et aussi de leurs parents sauvages présents dans leur environnement naturel ». ${ }^{4}$

2 Entre 2008 et 2014, nous avons étudié la formation et le déploiement de ce programme à travers une série d'entretiens menés à intervalle régulier avec ses principaux coordinateurs, différents experts ayant contribué à son dessein et sa mise en œuvre avec des paysans bénéficiaires au niveau national, régional et local ${ }^{5}$. Il s'agit donc de suivre une politique publique depuis sa conception jusqu'à sa mise en œuvre sur le terrain, c'est-à-dire à la fois « par le haut de l'État » et « par le bas ». Du fait de son objet peu stabilisé (l'agro-biodiversité) et son caractère hybride, entre politiques de conservation de l'environnement et politiques agricoles, le PROMAC revêt une dimension largement expérimentale qui nécessite la construction de cadrages et la mobilisation de savoirs nouveaux pour l'administration mexicaine. De ce fait, c'est un objet particulièrement pertinent pour étudier comment différents types de savoirs sont mobilisés ou au contraire marginalisés dans une politique publique, et ce dans l'objectif de rendre un objet gouvernable.

3 Cette politique de conservation de l'agro-biodiversité peut être considérée comme caractéristique d'une science based policy au sens où elle a fait appel pour sa mise en œuvre, à un effort de systématisation des données sur le maiis et à un appareillage cartographique et statistique. Cet article s'intéresse précisément au rôle des savoirs scientifiques dans le PROMAC et défend l'idée que s'ils sont déterminants dans le but de ce programme, leur importance décline tout au long de sa mise en œuvre. Au fur et à mesure du processus de traduction des savoirs scientifiques en politique publique, on assiste à une lente "érosion" de ces savoirs face aux contraintes politiques et administratives qui pèsent de manière beaucoup plus structurelle sur la mise en œuvre du programme. Ainsi, on peut se demander dans quelle mesure l'expertise scientifique ne sert pas plus à rendre un objet gouvernable, c'est-à-dire insérable dans l'appareil étatique, qu'à le gouverner effectivement. Autrement dit, l'expertise scientifique sert plus à délimiter et caractériser une problématique pour pouvoir l'inscrire dans les actions du gouvernement qu'à traiter un problème effectivement.

4 À un niveau théorique, on pourra s'appuyer sur différents travaux qui croisent sociologie des sciences (Pestre 2006, Hackett et al. 2008, Bonneuil \& Joly 2013) et sociologie de l'action publique (Padioleau 1982, King \& Le Gallès 2011, Lascoumes \& Le Gallès 2012) pour montrer différents rôles et usages des savoirs dans les politiques publiques (Haas 1989, Hope 1999, Jasanoff \& Martello 2004, Roger 2010, Dahan \& Guillemot 2015, Chauvet 2015). Nous nous appuierons en premier lieu sur le concept de transcodage proposé par Pierre Lascoumes au milieu des années 90 dans son livre L'écopouvoir (Lascoumes 1994). Lascoumes proposait d'adapter à la sociologie politique et, plus particulièrement, à l'analyse des politiques publiques, la sociologie de la traduction (Callon 1986, Akrich et al. 2006). La notion de transcodage a ainsi permis de mettre en lumière les déplacements de sens, de logiques et d'actions entre différentes parties prenantes (médias, société civile, instances gouvernementales) dans la gouvernance d'un problème public (en l'occurrence, les problèmes environnementaux), mais également au sein même de l'administration. Si cette notion est particulièrement utile pour comprendre comment certains savoirs scientifiques peuvent contribuer au cadrage général d'une politique publique, elle ne s'applique pas à la seule analyse du passage de la sphère cognitive à celle de l'action publique. La notion permet également 
de montrer comment, dans la création d'une nouvelle politique publique, ces nouveaux savoirs doivent s'insérer dans le cadre des institutions et des pratiques qui lui préexistent au sein de l'administration, avec les effets de torsions et de dilutions que ce déplacement suppose.

Dans l'optique de suivre ces chaînes de traduction, dans un premier temps, nous revenons sur la manière dont le problème de l'agro-biodiversité des maïs mexicains a été rendu lisible dans la sphère institutionnelle. Nous allons analyser comment les objectifs et les méthodes du PROMAC s'insèrent dans l'appareil administratif mexicain (Tableau 1), avec les difficultés que cela pose. Nous verrons ensuite comment, face à ces difficultés, deux évaluations contradictoires s'opposent sur la question de la nécessité d'une réforme de fond du programme. Enfin, nous donnerons une image contrastée du PROMAC depuis le niveau intermédiaire des aires protégées où il est censé opérer et depuis la vision de ceux qui sont censés en bénéficier: les paysans mexicains qui cultivent les maïs natifs.

Tableau 1 : Institutions mexicaines actives sur le thème de la conservation des maïs et de la biosécurité

\begin{tabular}{|c|c|c|}
\hline AUTORITES ENVIRONEMENTALES & $\begin{array}{l}\text { AUTORITES INTERMINISTERIELLES de } \\
\text { BIOSECURITE }\end{array}$ & AUTORITES AGRICOLES \\
\hline $\begin{array}{l}\text { SEMARNAT (Secretaria de Medio Ambiente y } \\
\text { Recursos Naturales) }\end{array}$ & \multirow{4}{*}{$\begin{array}{l}\text { CIBIOGEM (Comisión Interministerial de } \\
\text { Bioseguridad y Organismos Genéticamente } \\
\text { Modificados) }\end{array}$} & \multirow[t]{4}{*}{$\begin{array}{l}\text { SADER (Secretaria de Agricultura y Desarrollo } \\
\text { Rural) }\end{array}$} \\
\hline $\begin{array}{l}\text { CONANP (Comision Nacional de Areas } \\
\text { Naturales Protegidas) }\end{array}$ & & \\
\hline $\begin{array}{l}\text { CONABIO (Comisión Nacional para el } \\
\text { conocimiento y uso de la Biodiversidad) }\end{array}$ & & \\
\hline $\begin{array}{l}\text { INECC (Instituto Nacional de Ecología y } \\
\text { Cambio Climático) }\end{array}$ & & \\
\hline
\end{tabular}

\section{Mettre en œuvre un nouveau programme : mobiliser les experts pour une « science based policy »?}

6 La mise en œuvre d'une politique de conservation de l'agrobiodiversité des maïs a impliqué, à différents stades et plus ou moins directement avec des scientifiques issus de disciplines différentes et ayant travaillé sur le thème du maïs. Ces scientifiques forment une très large communauté aux contours flous et réunissant des communautés épistémiques différentes. Cette partie explore comment ces différentes communautés d'experts ont été mises à contribution pour dessiner un programme et comment, dès l'amont du programme, les connaissances mobilisées sont soumises à des contraintes institutionnelles et administratives.

\section{Rendre le problème lisible, quoi conserver et où ?: le Proyecto Global de Maices Nativos}

7 En amont de la mise en œuvre du PROMAC, un important projet de systématisation d'informations et de génération de nouvelles données sur le maïs a été mis en œuvre entre 2004 et 2011. L'enjeu était de mettre à jour la distribution du maïs criollo ${ }^{6}$ sur toute la république, selon les centres d'origine et de diversité du maïs (Kato et al. 2009), et établir, ainsi, sur cette base, les politiques de conservation et les zones d'intervention. 
8 Dans le cadre de ce projet appelé Proyecto Global de Maices Nativos (PGMN), différentes recherches ont ainsi été commandées à des équipes de scientifiques. Ces études ont été dirigées depuis l'institution publique en charge de la connaissance et de la mise en valeur de la biodiversité mexicaine - la CONABIO (Comisión Nacional de uso y aprovechamiento de la Biodiversidad) - et coordonnée par la principale institution de recherche agronomique du pays, l'INIFAP (Instituto Nacional de Investigaciones Forestales Agricolas y Pecuarias). La ligne de travail la plus importante si l'on considère le nombre de scientifiques et techniciens impliqués (plus de 200 chercheurs de quelques 65 institutions) et l'argent investi, a pris la forme d'un grand projet de collectes des maïs locaux et des parents sauvages : le Teocintle et le Tripsacum. Cette collecte historique a nécessité la participation des principales institutions agronomiques nationales et régionales (INIFAP, Université Chapingo, Centro de Investigación Científica de Yucatán, Universidad Autónoma de Nuevo León, Universidad Autónoma de Sinaloa...). Une cartographie plus précise et actualisée de la diversité des maïs et de ses parents sauvages, avec la découverte de nouvelles variétés de Tripsacum, a été constituée (CONABIO 201, 2020)7. Cet effort a donné lieu à une géographie du maïs plus précise et actualisée par rapport aux autres deux grandes collectes des années 1943-1954, 1968-1979 (Perales \& Golicher 2011 et 2014). Les données des collectes ont été aussi mises en relation avec le contexte agricole, social, économique et culturel montrant des nouvelles relations entre diversité de maïs et régions agricoles (Lazos \& Chauvet 2011). Si les résultats de ces études montrent que la répartition de la diversité n'a pas substantiellement changé, ils mettent aussi en évidence que certaines régions mexicaines, tel que le Nord du Pays, jusqu'ici considérées comme relativement pauvres en agrobiodiversité, s'avéraient également très riches en diversité de maïs (Lazos \& Chauvet 2011, CONABIO 2011, 2020, Perales \& Golicher 2011 et 2014). Si le projet a impliqué la mobilisation de compétences scientifiques très différentes (agronomie, cartographie, informatique, ethnobotanique, anthropologie...) on doit noter une très nette domination des approches agronomiques classiques et naturalistes dans la manière d'appréhender la question de la diversité des maïs (Gérard 2014). Si les sciences sociales autour du maïs sont mobilisées, ce n'est en effet qu'à la marge. Le questionnaire qui accompagnait les collectes portait essentiellement sur des données agronomiques (type de maïs, caractéristique du terrain, productivité, mode de culture...) et les informations socio-économiques qui ont été collectées (âge du cultivateur, appartenance ethnique, nom commun du maïs) ne permettaient pas vraiment d'appréhender la culture du maïs dans toute sa dimension sociale. Le seul projet de science sociale financé dans le cadre du PGMN a consisté à analyser ces données (Lazos \& Chauvet 2011). Elena Lazos et Michelle Chauvet ont souligné, dans ce numéro également, les limites, en termes de questionnement scientifique ainsi que de complétude, des données collectées. L'essentiel du projet a donc consisté, dans la tradition agronomique mexicaine, à collecter et établir un inventaire et une cartographie précis des différentes races et variétés de maïs, selon des critères essentiellement morphologiques et agronomiques (Sanchez Gonzalez 2011). Le PGMN correspond donc à une forme d'hybridation entre science et politique où la mobilisation d'experts, en l'occurrence majoritairement des agronomes, informaticiens et cartographes, permet de rendre le problème lisible (Lascoumes 1996, Scott 1998), c'est-à-dire compréhensible par les institutions publiques, étape essentielle pour le rendre gouvernable. Pourtant, si le projet semble avoir contribué indéniablement par ces financements à redynamiser la recherche sur le maïs, son impact pour rendre la question concrètement gouvernable, on va le voir, est moins évident. En effet, alors 
même qu'il semblait explicite dans les articles 86, 87 et 88 de la Ley de Bioseguridad y Organismos Généticamente Modificados (LBOGM) de 2005, que la cartographie de la distribution des races de maïs (Figure 1) devait servir de base scientifique à la définition de zones de conservation, les données produites dans ce projet, notamment au niveau cartographique, n'ont pas vraiment été mises à contribution au moment de la mise en place du PROMAC (Perales 2017).

Figure 1 : Distribution des races de maïs natif sur le territoire mexicain. Source : Distribución de maíces nativos en México. CONABIO 2015

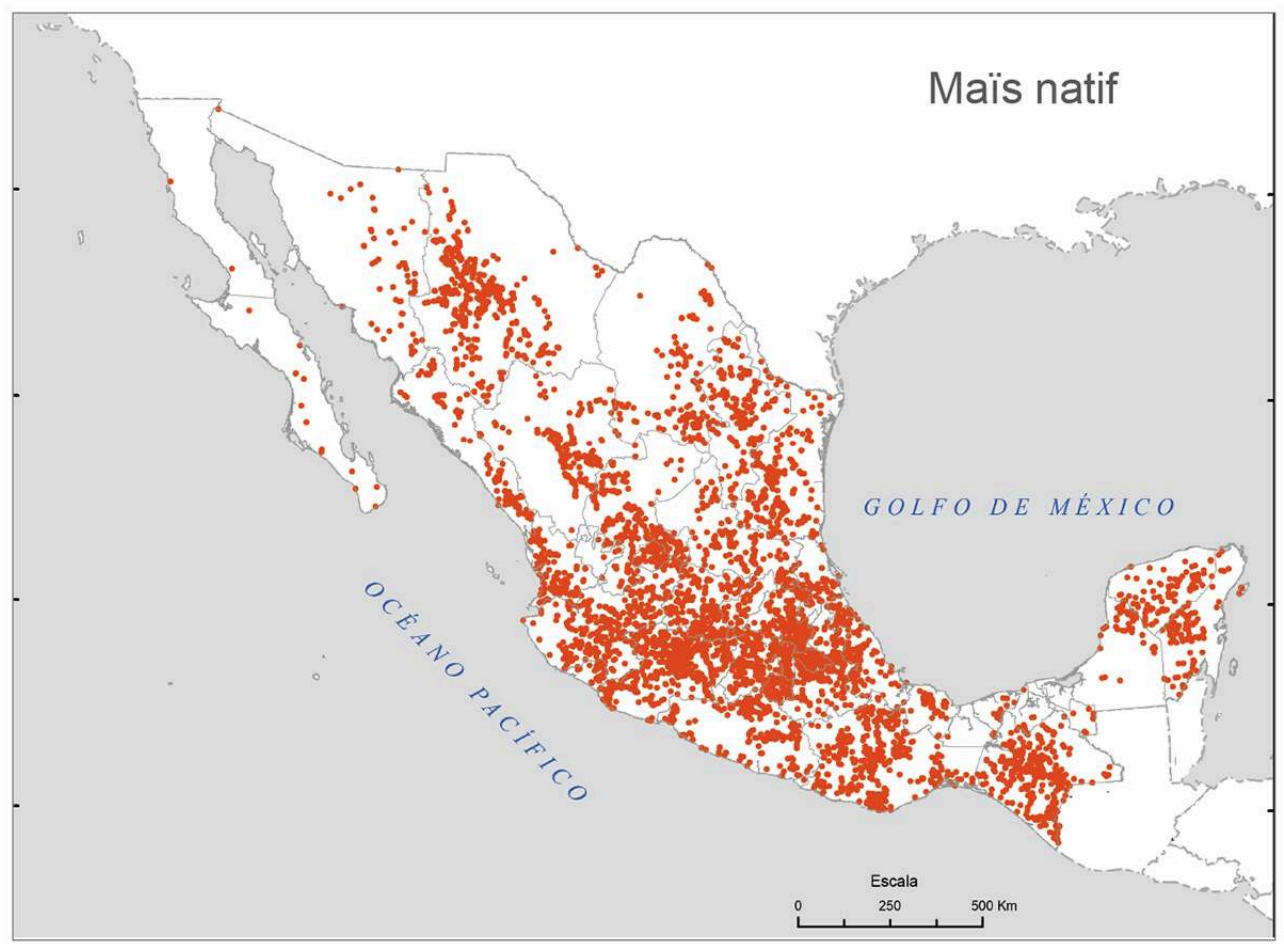

\section{Mettre en place le PROMAC : temporalités, compétences, méthodes}

Parallèlement à cet effort de compilation et d'actualisation des données et sans en attendre les résultats, dès 2008 , le ministère de l'environnement décide de mettre en place dans l'urgence son propre programme de conservation des maïs natifs. Il existe donc une certaine superposition temporelle entre la séquence de mise en lisibilité du problème et celle de son traitement politico-administratif. Pour le dire plus clairement, la SEMARNAT se lance dans un programme de conservation des maïs locaux sans savoir exactement quels maïs sont le plus menacés par l'érosion génétique, ni, dans quelles zones intervenir, ni quelle méthodologie conserver. Cette urgence est notamment dictée par l'allocation d'importants fonds à la SEMARNAT pour répondre à l'injonction de conservation présente dans la LBOGM. Nous ne pouvons faire ici que des hypothèses concernant le déblocage de ces fonds et l'urgence apparente à mettre en œuvre un tel programme de conservation des maïs locaux : Volonté politique de traiter le problème de l'érosion de l'agrobiodiversité des maïs ? Stratégie « d'évitement du blâme » (Blame avoidance) (Weaver 1986) pour des institutions environnementales soucieuses d'apparaître comme les bons élèves de politiques de biosécurité? Volonté de rapidement mettre en place une contrepartie écologique à la libération des maïs 
transgéniques dans la perspective d'imposer le principe de la coexistence (Foyer \& Bonneuil 2015)? Simple opportunité financière (l'argent était disponible à ce moment) ? Ces hypothèses n'épuisent pas la question et ne sont surtout pas exclusives l'une de l'autre. Elles peuvent en effet correspondre à l'agenda de différents acteurs de l'appareil étatique mexicain. Toujours est-il que la mise en place du PROMAC est largement soumise aux contraintes temporelles de cet agenda défini dans l'urgence.

Au-delà de ces contraintes temporelles, une première contrainte institutionnelle importante concerne le choix de l'institution la mieux à même de mettre en œuvre ce programme au sein de la SEMARNAT. La CONABIO, qui avait mis en place le PGMC aurait pu, du fait de l'expertise qu'elle était en train de mobiliser, être choisie comme l'institution chargée de l'application de ce programme mais elle est historiquement plus spécialisée sur l'étude et la systématisation de données sur la biodiversité que sur la mise en œuvre de programme de conservation. Dans l'organigramme institutionnel de la SEMARNAT ${ }^{8}$, l'institution la mieux à même d'opérer ce type de programme est la Comision Nacional de Areas Protegidas (CONANP) qui, à travers des programmes comme les PRODERS notamment (Dumoulin 2003, Léonard et Foyer 2011), a acquis depuis la fin des années 90 une certaine expérience quant à la mise en œuvre de normes de conservation et de projets de développement durable en partenariat avec les populations locales. Ce choix de la CONANP, s'il semble logique d'un point de vue institutionnel, pose d'emblée deux contraintes majeures pour la conservation des maïs locaux : une contrainte de compétence territoriale et une autre par rapport à la qualification et à la culture administrative de son personnel. La première contrainte renvoie au fait que la CONANP n'a vocation à opérer via son personnel local que dans les différentes aires naturelles protégées (ANP) (réserves de la biosphère, parcs naturels...) dont elle a la responsabilité (Figure 2).

Figure 2 : Les aires naturelles protégées fédérales du Mexique. Source : Áreas Naturales Protegidas Federales de México. CONANP 2016

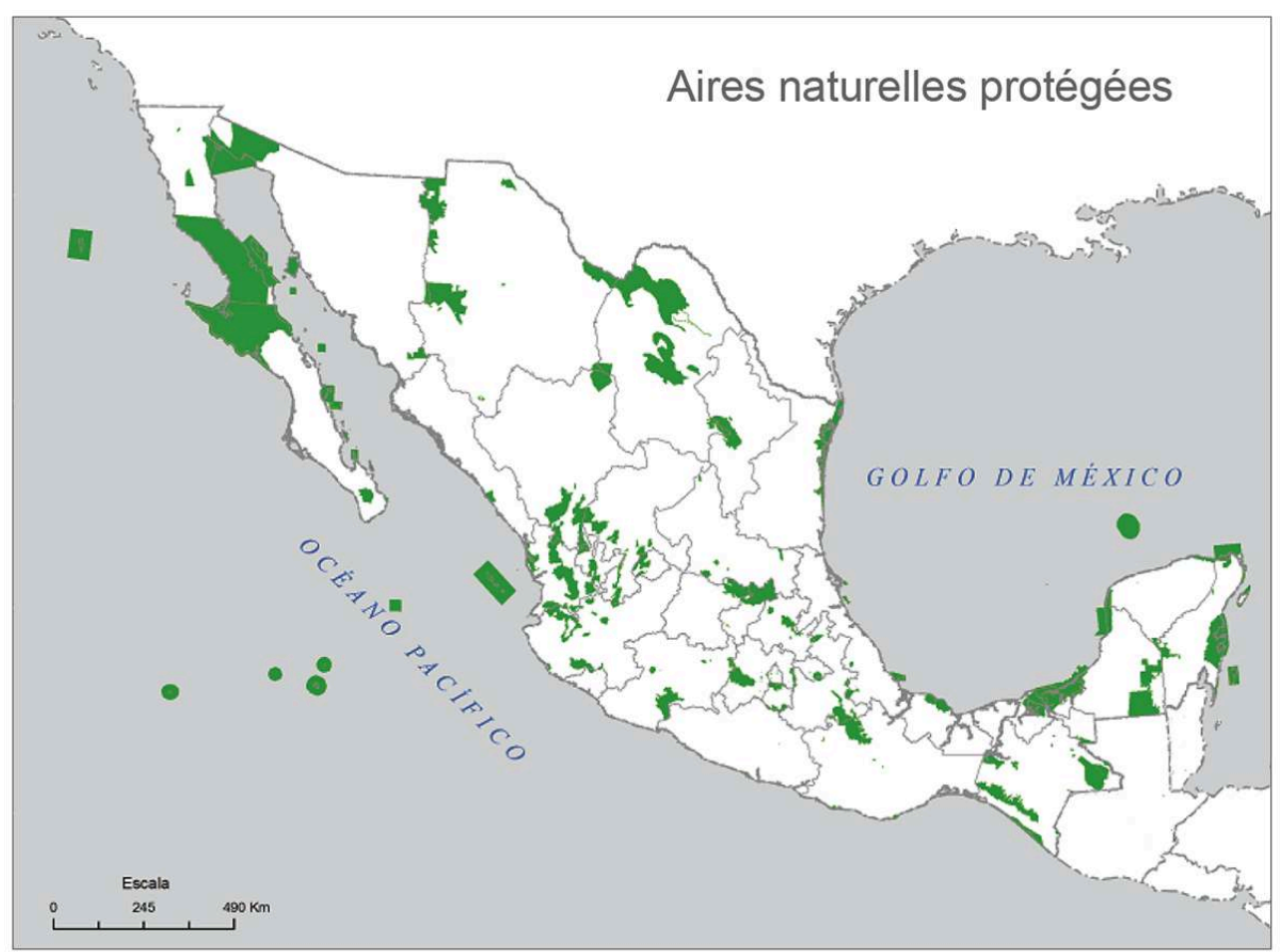



comme à haute diversité de maïs natifs et/ou en danger, ni avec les plus importantes régions où l'on pratique une agriculture paysanne. Cela signifie concrètement que le PROMAC ne pourra opérer que dans les zones des Aires Naturelles Protégées où les activités agricoles sont autorisées - par exemple dans les zones tampons des réserves de la biosphère- ce qui limite drastiquement son aire d'application par rapport aux zones où la conservation des maïs locaux pourrait représenter un enjeu écologique. Les principales zones où les maïs natifs sont cultivés se trouvent de facto largement exclues de la sphère territoriale de compétence du PROMAC.

13 La deuxième contrainte concerne la compétence thématique de la CONANP et la formation de son personnel. Dans cette institution domine un personnel scientifique formé majoritairement en biologie et en écologie sur des questions de conservation de la biodiversité non agricole. Même si la culture de ces institutions a évolué avec la prise en compte des activités humaines, dans cette approche conservationniste, les activités agricoles peuvent être pensées de manière conflictuelle avec ce qui reste le cœur de métier de la CONANP : la conservation des aires naturelles protégées. Pour pallier ce manque de connaissance sur les questions agronomiques concernant la partie scientifique et technique de sa mise en œuvre, on recrute un jeune agronome formé en agro-écologie à l'université de Chapingo et travaillant sur des questions de conservation de l'agrobiodiversité des maïs dans une organisation civile de l'État du Michoacan. Son idée initiale est de s'inspirer des différents programmes liés aux techniques de conservation participative in situ des maïs locaux et il convoque une réunion des principaux spécialistes de cette communauté à la fin de l'année 2008. La définition de la conservation in situ a changé entre-temps. Dans les années 1970, elle renvoyait à la conservation des variétés natives dans leur habitat agricole naturel sans prendre en compte les processus d'évolution des variétés et d'interaction avec les pratiques paysannes. C'est seulement à partir des années 1980 que ces aspects sont intégrés dans la définition et les objectifs de l'in situ (Altieri \& Merrick 1987, Louette et al. 1997, Brush 2000, Bellon 2004, Jarvis et al. 2011, De Boef et al. 2013). Si au niveau théorique, la définition de l'in situ continue à s'enrichir, il reste encore un cadre conceptuel très rigide qui rend mal compte de la complexité du monde du maïs natif qui peut changer ou se transformer en fonction de facteurs naturels (aléas climatiques, flux génétique, perte de semences), sociaux (systèmes complexes de circulations de semences, pratiques agricoles) ou économiques (programmes gouvernementaux, adaptation à la demande). Face à cette complexité, la conservation in situ a été considérée par certains scientifiques mexicains comme un ensemble d'initiatives visant à assurer la continuité du processus évolutif des variétés de maïs au sein des pratiques paysannes plutôt qu'un dispositif de conservation de matériel génétique "stable » (Fernando Castillo et Rafael Ortega Paczka, entretien personnel, 25/03/2008; Hugo Perales Rivera, entretien personnel, 05/09/2011). Dans cette optique, les projets in situ ainsi que les programmes de sélection du maïs doivent être analysés comme des activités institutionnelles visant la conservation et l'usage des variétés plus adaptées aux besoins de l'agriculture paysanne. Ces deux volets devraient donc opérer en concert et se soutenir mutuellement. Si les savoirs et techniques de la conservation in situ semblent a priori cadrer parfaitement avec les objectifs du PROMAC, leur traduction en termes de politiques publiques semble d'emblée plus problématique. En effet, les agronomes impliqués au Mexique dans des projets de conservation in situ et de sélection participative sont peu nombreux, une dizaine à peine ayant une expérience de 
long terme de ce type de pratiques, ce qui pose d'emblée un problème de compétences mobilisables. Ces projets sont menés de manière très localisée, sur quelques parcelles au sein des communautés avec lesquelles les chercheurs ont établi des collaborations et des relations de confiance sur le long terme. Cet aspect pose évidemment question quant à la possibilité de reproduire ce type d'expériences dans le cadre d'un programme d'ampleur national. Du fait de la spécificité des savoirs et techniques mobilisées et du suivi quasi personnalisé que la conservation in situ nécessite, celle-ci ne semble d'emblée que difficilement extrapolable à l'échelle du pays. Néanmoins la réticence principale des agronomes de la conservation in situ à s'impliquer dans le dessein des lignes du projet concerne le principe, alors envisagé, d'un appui financier direct (subvention ou paiement pour service environnemental) en faveur des paysans qui sèment les maïs natifs (Garibay 2012). Les risques de clientélisme, de corruption, de division ou de manque de suivi en cas d'arrêt des financements, sont pointés comme les principaux risques possibles dans les programmes d'appuis financiers en direction du monde rural mexicain. Malgré ces réserves des spécialistes de la conservation in situ et du fait des contraintes temporelles imposées par l'agenda politique, le PROMAC est conçu avant tout comme un programme de "paiement pour conservation in situ " qui s'apparente à une subvention agricole classique où les paysans reçoivent une soixantaine d'euros (1200 pesos) à l'hectare pour planter une variété de maïs natif listée comme à risque d'érosion. Du point de vue de la gestion du projet, la priorité à ce dispositif présente également l'avantage d'être opérationnel rapidement par rapport au but d'autres types d'actions plus spécifiques et, surtout, de faciliter le décaissement rapide de presque trois millions d'euros (60 millions de pesos) alloués au programme pour sa première année d'opération en 2009. En complément de ces paiements directs, mais dans une proportion du budget bien moindre, le programme vise à financer également d'autres types d'actions comme des études sur la diversité des maiis, des formations à l'agro-écologie, la constitution de banques locales de semences ou encore des foires de semences et des projets de transformation du maïs en produits alimentaires de terroir. On va voir néanmoins que ce choix entre paiement direct et actions plus spécifiquement tournées vers l'agro-écologie des maïs mexicains va constituer un point de clivage durable dans la manière de concevoir le programme. D'emblée, la tentative de transcodage des perspectives de la communauté des agronomes de la conservation in situ dans les termes d'une institution spécialisée dans la conservation de la biodiversité dans les aires naturelles protégées, s'avère problématique et les savoirs scientifiques doivent se plier aux contraintes politiques, institutionnelles et administratives.

14 En ce qui concerne l'articulation du PGMC et du PROMAC on voit bien que la séquence mécanique d'une science based policies où une expertise rendrait le problème lisible dans un premier temps pour commencer à projeter des dispositifs rationnels dans un second temps, ne correspond pas à la réalité. Non seulement les séquences se superposent dans le temps, mais on a vu également qu'il existe un déphasage entre les principaux résultats du PGMC (la zonification précise des centres de diversité) et le périmètre d'intervention du PROMAC (les aires naturelles protégées). Dès la phase de dessin du PROMAC, on comprend donc que les différents types de savoirs mobilisés ne déterminent qu'à la marge la mise en gouvernabilité de la conservation des maïs mexicains. Cette tendance à la mobilisation marginale des savoirs au profit d'une logique gestionnaire et administrative va se confirmer dans les suites du programme et à différents niveaux. 


\section{Logique gestionnaire VS logique socio-environnementale}

\section{L'approche gestionnaire pour une subvention agricole à la petite paysannerie}

15 La gestion administrative et financière du projet est confiée au directeur des activités productives alternatives de la CONANP, formé comme ingénieur agronome en zootechnique à l'Université de Chapingo et titulaire d'un master en économie. Outre la responsabilité du PROMAC, celui-ci est chargé de la gestion de cinq autres programmes de la CONANP combinant des objectifs environnementaux et productifs'. Parmi ces autres programmes, le PROMAC est un des moins importants en termes budgétaire. Initialement, en 2009, le programme avait été doté d'un financement de 60 millions de pesos. Son budget a été ensuite divisé par deux et le programme a fonctionné avec 30 millions de pesos jusqu'à 2012, puis partir de 2013, le budget a encore été réduit à environ 19 millions de pesos. (PROMAC 2017). La priorité de ce responsable administratif est en premier lieu de décaisser une partie, la plus importante possible du budget alloué, pour que le programme ne soit pas menacé de suppression et ensuite, de faire en sorte que les subsides arrivent directement aux paysans, sans que l'argent soit capté par différents intermédiaires comme c'est le cas dans d'autres programmes. Dans cette optique, il privilégie le mécanisme de paiement pour conservation in situ, en premier lieu, car il a l'expérience des démarches institutionnelles auprès du ministère $\mathrm{du}$ budget pour mettre en œuvre ce type de subventions. Cette justification par l'expérience institutionnelle peut renvoyer tout autant à une forme de facilité (moindre contrainte) qu'à une forme de réalisme politique (meilleure chance de réussite). Elle révèle en tous cas l'importance des habitus institutionnels dans la mise en œuvre des politiques publiques. Outre cette justification, le gestionnaire du programme évoque également le fait qu'en s'adressant aux petits paysans généralement exclus des grands programmes modernisateurs de la SADER, le PROMAC rétablit une certaine forme d'équilibre. Se référant notamment à l'un des programmes les plus importants de SADER, le PROCAMPO, il explique ainsi : " on paie bien des subventions à des gens beaucoup plus riches qui ont beaucoup plus d'entrées et de ressources et qui sèment des tomates dans le Sinaloa et qui touchent la majorité des subventions!» (Jose Juan Arriola Royo, entretien personnel, 26/9/2013). Dans cette optique, son objectif est donc que le PROMAC demeure avant tout un programme d'appuis directs aux paysans et que ces appuis représentent près de $80 \%$ du budget total du projet, notamment par rapport aux autres modalités d'intervention (ateliers de formations, études, organisations de ferias...) qu'il juge beaucoup plus compliqué à gérer d'un point de vue comptable et administratif.

16 Cette modalité de fonctionnement est celle qui va s'imposer les premières années de fonctionnement du programme et qui a prédominé jusqu'en 2015. Elle est validée et légitimée par différentes initiatives d'évaluation du programme dès la première année, puis en 2012. Ces évaluations menées par des organes d'audit externe sont intéressantes car elles ne s'appuient en aucun cas sur une expertise agronomique par rapport à la conservation des maïs ni sur des enquêtes auprès du public-cible de ces programmes. L'approche privilégiée par ces évaluations est essentiellement gestionnaire au sens où ce qui est validé, c'est avant tout le respect des normes légales, administratives et budgétaires (Instituto para la gestion integral de cuencas hidrograficas 2009) ainsi que la portée du programme, évaluée en terme quantitatif 
bien plus que qualitatif. Basée sur la méthodologie de la CONEVAL (Comision Nacional de Evaluacion de la Politica de Desarrollo Social), l'évaluation de 2012 s'intéresse ainsi au dessein, à la planification, à la couverture, à la focalisation, à la mise en œuvre, à la perception de la population cible et enfin, à la mesure des résultats du programme, ceci de manière avant tout technocratique. Sur ces critères, l'évaluation externe de 2012, d'un coût de 900000 pesos, s'avère extrêmement positive puisqu'elle conclue que

«Le programme est cohérent et les résultats au bout de trois ans d'opération permettent de voir que sa mise en œuvre a été un succès car ont été conservées 44 des 60 races ou variétés de maïs, [...], et qu'ont été appuyées 64.500 personnes des communautés rurales et/ou indigènes. » (CONEVAL 2012).

17 Cette légitimation d'une approche gestionnaire basée sur le dispositif de paiement pour conservation in situ est d'autant plus importante qu'elle est alors mise à l'épreuve par une critique interne et un cadrage concurrent venant d'une nouvelle instance de direction du programme.

\section{L'impossible socialisation du PROMAC}

18 En 2010, un coordinateur national du programme est recruté par la direction de la CONANP avec pour mission explicite de donner au programme un caractère plus social. De par sa formation, Ricardo Garibay présente un profil multidisciplinaire puisque sa discipline initiale est l'anthropologie, il est titulaire également d'une maîtrise en développement rural et poursuit alors un doctorat en sciences naturelles. Il avait pu se familiariser aux problématiques liées au maïs natif à travers plusieurs terrains d'étude à Yaxcaba, la communauté yucatèque qui a hébergé, de 1979 á 1990, le projet pionnier « Dynamique de la Milpa » dirigé par l'un des plus grands ethnobotanistes et spécialiste du maïs mexicain, Efrain Hernandez Xolocotzi. Ricardo Garibay bénéficie également d'une solide expérience institutionnelle puisqu'il a travaillé dans différentes institutions et différents programmes du ministère de l'environnement depuis une vingtaine d'année, notamment à la tête d'un programme de foresterie communautaire relativement novateur dans sa volonté d'articuler activités productives, développement communautaire et conservation: le Programa de Desarrollo Forestal Comunitario (PROCYMAF). Ce profil Daest typique de ce que l'on peut appeler «l'environnementalisme social mexicain" (Foyer \& Dumoulin 2015), à savoir un mélange de formation universitaire et d'expériences dans des projets de développement depuis la société civile et/ou le gouvernement, avec une approche plus ouvertement politique, et moins biologisante de l'écologie, que ne l'est l'approche conservationniste. Son appartenance à ce monde de l'environnementalisme social se reflète notamment dans l'une de ses premières actions: la refonte du groupe d'expertise qu'il souhaite désormais mobiliser pour réorienter le PROMAC. Il décide en effet de rééquilibrer ce groupe au profit des sciences sociales, notamment l'ethnobotanique et l'anthropologie. En plus des premiers agronomes associés au programme, il s'appuie aussi sur différents chercheurs (Eckart Boegue-INAH, Victor Manuel Toledo-UNAM, Narciso Barrera-UNAM, Marta Astier-UNAM, Elena LazosUNAM...) appartenant à la Red de Etnoecología y Patrimonio Biocultura ${ }^{10}$ et sur des experts issus d'organisations civiles spécialisées dans le développement communautaire (GEA, ERA). Dans la perspective de cette communauté épistémique, l'accent est mis sur le couplage entre diversité biologique et diversité culturelle, en insistant notamment dans le contexte mexicain sur les liens entre populations indigènes et biodiversité et sur la 
nécessité d'appuyer les dynamiques de développement sur le tissu des organisations communautaires. Au moins autant qu'à donner son opinion sur le programme et les opportunités de réorientation, il semble évident que ce groupe d'assesseurs permet de légitimer la stratégie de réorientation du programme. Dans un rapport qui présente les principales lignes de réorientations, Garibay explique :

«J'assume la responsabilité de ces propositions mais je tiens à souligner également que je me fais le porte-voix des opinions de ceux que j'ai interviewés (Directeurs des ANP, fonctionnaires et bénéficiaires du programme) et qui coïncident à $90 \%$ sur la nécessité de faire des modifications substantielles au programme » (Garibay 2010)

19 Un des premiers constats, ressortant du bilan critique mené par Garibay, est que le programme a été dessiné sur le modèle des programmes assistentialistes destinés aux populations rurales mexicaines. Ainsi, à la domination du dispositif de «paiement pour conservation in situ» devait répondre un rééquilibrage du budget du programme au profit des autres lignes d'action comme l'organisation d'ateliers de formation à l'agroécologie, le soutien à des évènements comme les fêtes du maïs ou la mise en place de réseau d'échanges de semences. Ainsi, en 2011, le budget consacré au paiement direct ne représentait plus que $50 \%$ du budget total du programme (Garibay, entretien personnel, 18/08/2012), les paiements directs étant désormais conditionnés à l'articulation avec les autres lignes d'action, dans une approche plus globale de la conservation des maïs. Une autre critique structurelle concerne les limites imposées par la marge financière et territoriale imposée par la CONANP, marge avec laquelle il faut composer pour exécuter le programme (Garibay 2010). Il a été estimé qu'actuellement, du fait de son budget limité et de son périmètre d'action, le Programme ne touche qu'environ $1 \%$ des producteurs de maïs indigènes, son impact est donc très limité par rapport à la conservation qui existe de facto (Perales 2017). Cette dernière évaluation du programme confirme en outre que les activités les plus appréciées dans le PROMAC par les agriculteurs ont été les formations, les foires et autres activités sociales. Cependant le programme a été adapté pour fonctionner d'un point de vue administratif et, au lieu d'avoir un impact dans la conservation, il se «superpose" aux dynamiques agricoles du mais criollo (Perales 2017). Considéré comme programme d'aide directe, ses moyens financiers limités doivent être mis en perspective avec le budget de programme tel le Programme de maïs et haricot (Programa de Maíz y Frijol, PROMAF) que promeut alors la SADER pour l'adoption de paquets technologiques (et donc de semences hybrides) et qui opérait en 2011 avec un budget de 1,1 million de pesos, soit près de 40 fois celui du PROMAC. Cette asymétrie budgétaire touche également un troisième point fondamental de critique porté par les experts : celui du manque de cohérence dans les politiques publiques mexicaines envers le monde rural, politiques publiques dont les objectifs peuvent être totalement contradictoires comme, on vient de le voir, entre le PROMAC et le PROMAF.

Toutes ces lignes convergent donc vers l'objectif de « socialiser » le PROMAC selon une perspective plus intégrale qui articule les objectifs de conservation avec ceux du renforcement des capacités dans les communautés, en s'appuyant sur différentes alliances au sein du monde académique et de la société civile. Ce projet de réorientation semble être sur le point d'être mis en œuvre à la fin de l'année 2012 quand le coordinateur national négocie un budget supplémentaire de 18 millions de pesos auprès des instances de la CONABIO. Ce budget devait être consacré à la mise œuvre de cette dynamique de socialisation. Cependant, la remise en cause radicale de l'évaluation de la CONEVAL par l'approche plus qualitative de Garibay marque un point de rupture 
entre la direction administrative du programme et la direction plus politique. Au-delà du conflit de personne quant à la direction du programme, c'est un schisme quant à la vision même de ce dernier, entre, d'une part, l'approche gestionnaire-budgétaire et, d'autre part, l'approche plus socio-environnementale qui est arbitré par la direction de la CONANP au profit de la première. Le coordinateur général du programme est remercié et les 18 millions de pesos constituent un fond géré par la CONABIO. Cette institution organise début 2014 un nouvel atelier avec différents spécialistes du maïs pour essayer de recenser, d'appuyer et de diffuser différentes success stories autour du maïs au Mexique. Cet atelier marque la volonté d'impliquer de nouveau les agronomes de la conservation in situ dans un compromis entre cette communauté épistémique et celle de la diversité bio-culturelle. Les nouvelles modalités de fonctionnement du PROMAC semblent donc être celles d'un sous-programme géré par la CONANP et qui fonctionne essentiellement sur la modalité des paiements pour conservation in situ et, pour une part budgétaire moindre, un fond géré par la CONABIO censé appuyer des actions complémentaires comme les foires, la sélection participative, les banques communautaires ou encore les marchés alternatifs de produits dérivés du maïs.

Dans cette partie, on a vu que différentes formes d'hybridations de savoirs/politiques s'opposent dans la conduite du programme (Tableau 2).

Tableau 2 : Les communautés épistémiques en concurrence dans la mise en œuvre du PROMAC

\begin{tabular}{|c|c|c|c|}
\hline Communauté épistémique & Gestion & Conservation in situ & Diversité bio culturelle \\
\hline Disciplines & $\begin{array}{l}\text { Économie/gestion/ } \\
\text { agronomie }\end{array}$ & $\begin{array}{l}\text { Agro-écologie, Agronomie } \\
\text { Écologie des Populations }\end{array}$ & Ethnobotanique, Anthropologie \\
\hline Spécialités, compétences & $\begin{array}{l}\text { Maîtrise des cadres } \\
\text { légaux et institutionnels }\end{array}$ & $\begin{array}{l}\text { Classification et suivi de l'évolution } \\
\text { des populations des maiis; distribution } \\
\text { géographique; recherche des modèles, } \\
\text { conservation in situ des maïs en lien } \\
\text { avec la sélection. }\end{array}$ & $\begin{array}{l}\text { Mise en évidence des liens entre } \\
\text { agro-biodiversité des maïs et } \\
\text { dimensions culturelles et sociales }\end{array}$ \\
\hline Cadrage du Promac & $\begin{array}{l}\text { Priorité au paiement pour } \\
\text { conservation in situ }\end{array}$ & $\begin{array}{l}\text { Critique des paiements pour } \\
\text { conservation in situ, renforcement de } \\
\text { l'expertise technique sur la conservation } \\
\text { des maïs, la sélection, les actions en } \\
\text { soutien des échanges des semences } \\
\text { et des connaissances sur le thème de } \\
\text { l'agrobiodiversité. }\end{array}$ & $\begin{array}{l}\text { Volonté de "socialisation " } \\
\text { en lien avec des initiatives de } \\
\text { la société civile. Élargir les } \\
\text { problématiques de conservation } \\
\text { aux non-spécialistes. Lien plus } \\
\text { direct avec la lutte contre les } \\
\text { OGM }\end{array}$ \\
\hline $\begin{array}{l}\text { Conseil et légitimation auprès de } \\
\text { quelles instances administratives? }\end{array}$ & $\begin{array}{l}\text { Direction des activités } \\
\text { productives alternatives } \\
\text { de la CONANP }\end{array}$ & $\begin{array}{l}\text { Techniciens du PROMAC + CONABIO } \\
\text { (coordination des ressources } \\
\text { génétiques et biologiques) }\end{array}$ & $\begin{array}{l}\text { Coordination générale du } \\
\text { programme CONANP }\end{array}$ \\
\hline
\end{tabular}

La première forme, de type gestionnaire, ne s'appuie pas tant sur un collectif d'experts scientifiques externes spécialistes des maïs que sur un savoir-faire administratif issu de l'expérience bureaucratique de la gestion d'autres programmes d'appui. Si cette approche gestionnaire ne recouvre pas à proprement parler une communauté épistémique au sens canonique, elle renvoie bien à des objectifs politiques (répondre aux cadres institutionnels et légaux, transférer des fonds à des populations marginalisées) et s'appuie sur un type de savoirs pratiques qui, s'ils n'ont que peu à voir avec le maïs, n'en sont pas moins cruciaux. Ce type de savoirs, d'un point de vue de la conduite du PROMAC, s'avère en effet bien plus déterminant que ceux plus intellectuels de la communauté épistémique? de la diversité bioculturelle, censés appuyer la refonte du programme. L'alliance stratégique recherchée avec cette communauté par le coordinateur national du PROMAC n'a en effet pas débouché sur le recadrage de cet organisme PROMAC et la perspective normative et socio-environnementale a été marginalisée puis recyclée dans une sorte d'annexe au PROMAC, sous la forme «d'actions complémentaires ». En effet, le retour de la CONABIO et des agronomes de la conservation in situ à partir de 2013 apparaît comme une forme de compromis au 
niveau cognitif, entre une approche naturaliste et une approche plus sociale, et au niveau politique, entre le dispositif de paiement pour conservation in situ et les actions sociales de conservation des maïs. Ce compromis semble en tous cas être la solution institutionnelle choisie par les instances environnementales mexicaines pour réorienter le programme sans le bouleverser complètement. Bien qu'inféodées aux logiques administratives, ces divergences à la fois cognitives et politiques semblent importantes dans le dessein et la conduite du programme. On va voir néanmoins qu'elles deviennent marginales au moment de sa mise en œuvre locale.

\section{Le PROMAC desde abajo « depuis le bas »: les savoirs sous contrainte administratives et matérielles}

En ce qui concerne sa mise en œuvre, le PROMAC « redescend » vers les communautés en passant les différents échelons administratifs de la CONANP. Le personnel comprend généralement un responsable du programme au niveau de l'ANP ou d'une région prioritaire de conservation et des techniciens, les assesseurs, spécifiquement en charge du lien direct avec les bénéficiaires du programme. Enfin, ce sont les paysans euxmêmes qui sont non seulement bénéficiaires, mais également acteur du PROMAC. À chacun de ces niveaux, nous allons voir comment les savoirs, censés originellement orienter le programme, sont soumis à toute une série de contraintes administratives et matérielles.

\section{Le PROMAC vu des Aires Naturelles Protégées : intéresser les paysans à la conservation}

Les coordinateurs régionaux ont des profils évidemment divers. Ils sont le plus souvent agronomes ou biologistes et certains ont une longue expérience des organisations civiles de développement communautaires et correspondent au profil de que l'on a appelé l'environnementalisme social. Comme au niveau national, ces coordinateurs régionaux sont généralement responsables des autres programmes de "conservation indirecte » de la CONANP, c'est-à-dire ceux qui ne touchent pas tant à la conservation classique per se (basée sur des inventaires biologiques, la surveillance d'activités potentiellement nuisibles à l'environnement et la préservation de zones sans activité humaine) qu'à la conservation en lien avec les activités productives ${ }^{11}$. Les responsables régionaux du programme ont donc le plus souvent en charge d'autres programmes de développement communautaire environnemental, comme le PROCODES, centrés sur d'autres activités productives que l'agriculture du maïs. Face à ces autres programmes, le PROMAC reste marginal, au moins d'un point de vue budgétaire. Cependant, pour les différents responsables, le PROMAC apparaît comme une opportunité pour renforcer les liens avec les communautés avec lesquels ils travaillent déjà. Le PROMAC a représenté une opportunité pour élargir leur thématique d'action à une sphère productive jusque-là négligée, mais néanmoins centrale pour les populations rurales qui vivent dans les réserves ou dans les zones connexes de la production du maïs.

Un des rôles principaux de ces coordinateurs régionaux est de gérer les lourdes procédures administratives qui encadrent le programme et d'aider ses potentiels bénéficiaires à remplir correctement les nombreux formulaires et respecter les nombreux prérequis pour pouvoir être accepté au sein du programme (avoir des droits formels sur la terre, faire partie d'une communauté éligible et d'un groupe de 
producteurs constitué, faire valider les maïs couramment semés comme natifs.). Un des coordinateurs expliquent ainsi :

«La charge administrative des programmes de subvention est très lourde. Elle consomme de 60 à $70 \%$ du temps des personnes qui les mettent en œuvre et qui sont chargées du suivi du programme car il faut faire les accords, il faut réunir tous les documents d'identité, remplir les formulaires...» (Ivan Ortiz Lopez, entretien personnel, 27/10/2011). compétences techniques et scientifiques au sein de la CONANP pour établir des critères de conservation sérieux. Par exemple, en ce qui concerne le travail d'identification et de validation des maïs natifs, on fait systématiquement appel à un prestataire de services externes, généralement issu de l'institution agronomique la plus proche et la plus compétente. précarité de la situation des assesseurs techniques du programme, chargés de la mise en œuvre au niveau des communautés. Généralement recrutés pour la période d'opération du programme, c'est-à-dire de six à huit mois dans l'année en fonction des ressources disponibles, ces assesseurs sont le plus souvent des jeunes tout juste sortis des écoles d'agronomie ou d'écologie locales. Non seulement ils sont loin d'avoir toujours les connaissances spécifiques sur les maïs locaux, mais la précarité de leur emploi et les très faibles salaires ne permettent pas d'ancrer leur travail dans la durée puisqu'à la moindre opportunité d'obtenir un emploie plus stable, ceux-ci ne reprennent pas leur activité d'assesseurs d'une année sur l'autre. Le remplacement des assesseurs est donc particulièrement important. Outre le travail administratif qui occupe, on l'a vu, l'essentiel de leur temps, les assesseurs sont chargés d'une importante activité de contrôle. Ils doivent notamment s'assurer que les variétés locales de maïs subventionnées correspondent bien à des variétés locales en danger d'extinction et non pas, par exemple, à des générations avancées d'hybrides, s'assurer que les paysans assurent un bon suivi des parcelles concernées par le programme. Ils doivent aussi vérifier que les pratiques agricoles, telles que l'usage des produits chimiques, soit conforme aux règles établies par les responsables locaux du programme mais leur pouvoir de contrôle effectif est limité. Certaines régions sont très difficiles à couvrir du fait de l'éloignement géographique et de la difficulté des terrains souvent montagneux et/ou forestiers. Certaines communautés éligibles au programme peuvent se trouver parfois à plus de cinq heures de route de l'administration. Dans ces conditions, certains assesseurs admettent ne pouvoir contrôler que de 10 à $30 \%$ des communautés où s'applique le programme et que ces mécanismes de contrôle restent largement sujets à la bonne volonté des paysans

«Quand tu dis aux gens « nous venons contrôler », c'est pareil que quand les soldats arrivent et disent « nous voulons voir les plants de marijuana ». Évidemment, on ne va pas t'emmener là où pousse la marijuana. Il nous arrive la même chose, les gens nous amènent là où c'est propre » (Eloy Uitz Chi, entretien personnel, 24/07/2012).

Malgré ces contraintes nombreuses, on peut noter un certain attachement des assesseurs à ce programme, du fait de ses objectifs de conservation et de sa mise en œuvre relativement plus serrée. Un assesseur chargé des régions des Vallées Centrale de l'État de Oaxaca compare ainsi le PROMAC avec sa version productiviste du ministère de l'agriculture, le PROMAF, dont il a été également assesseur. 
«Le PROMAC est bien meilleur du fait que l'on travaille avec des communautés qui ont une certaine conscience de la nécessité de conserver l'environnement et le germoplasme des maïs. Ce sont bien des gens qui sèment du maïs, des groupes plus réduits avec lesquels on peut plus facilement travailler vu qu'ils reçoivent déjà d'autres appuis de la CONANP. On travaille avec eux de façon plus organisée à la différence du PROMAF. J'ai travaillé un temps pour ce programme [PROMAF] et je me suis confronté à la problématique que tous ceux qui reçoivent la subvention ne sèment pas forcément du maïs et beaucoup d'entre eux reçoivent l'appui pour faire autre chose » (Ivan Ortiz Lopez, entretien personnel, 27/10/2011). régionaux et assesseurs) le PROMAC, bien que marginal en termes de financement représente un pas de plus dans la logique de couplage entre conservation et sphères productives. On voit également qu'à ces échelons administratifs, les questions de savoirs sur les maïs et de cadrage du PROMAC s'effacent au profit d'une gestion quotidienne soumise à toute une série de contraintes administratives (paperasserie, bureaucratie, gestion de personnel), financières (manque de ressources) ou matérielles (gestion des distances) qui pèsent beaucoup plus structurellement sur la mise en œuvre du programme.

\section{Le PROMAC vu des champs : stratégies d'appropriation paysanne d'une ressource}

Le PROMAC s'adresse donc à des paysans semant des variétés de maïs natifs dans ou à proximité de différents types d'aires naturelles protégées. Il s'agit le plus souvent d'une agriculture d'auto-subsistance ou semi-commercial, sur de petites surfaces n'excédant que très rarement 5 ha par personne et basée sur la culture de la milpa. Si cette expression renvoie dans l'imaginaire scientifique à un système complexe et supposé écologiquement efficace de cultures associées (généralement les maiss, haricots, tomates et courges ou courgettes) (Nations \& Nigh 1980, Toledo 2000, Boege 2008), il recouvre en réalité toute une gamme de pratiques et de techniques y compris la monoculture, l'utilisation de générations avancées d'hybrides, la mécanisation, l'utilisation d'intrants chimiques et l'écobuage. Les paysans qui reçoivent l'appui du PROMAC cultivent donc des variétés locales de maïs avec des modalités très diverses, dans des environnements qui vont des plaines tropicales aux pentes montagneuses. Il est particulièrement difficile de caractériser précisément le type de savoirs associés à ces très diverses manières de cultiver le maïs et sans doute même est-il plus judicieux de parler de praxis/pratique de la milpa tant ces savoirs sont difficilement dissociables et isolables, d'une part, d'imaginaires, et d'autre part, de pratiques très concrètes (Barrera \& Toledo 2005).

31 Nos entretiens avec différents groupes de paysans révèlent que le PROMAC est généralement perçu comme un programme relativement similaire aux programmes ruraux assistentialistes du gouvernement mexicain, notamment des autorités rurales. $\mathrm{Au}$ moins lors des premières années du programme, le PROMAC est souvent considéré comme une sorte de "PROCAMPO écologique ", c'est à dire un programme de soutien $\mathrm{du}$ gouvernement au monde rural, mais avec une teneur écologique. Ce soutien est diversement apprécié selon les zones; pour certains, il est largement insuffisant par rapport aux besoins, mais pour la plupart des paysans interviewés, c'est une aide précieuse car, bien souvent, un des rares programmes publics auxquels ils ont accès, les autres grands programmes des autres ministères (agriculture, santé, foresterie, Commission Nationale Indigène) étant pour beaucoup inaccessibles. Si le PROMAC ne

Revue d'ethnoécologie, Supplément 2 | 2021 
couvre pas forcément les dépenses ${ }^{12}$ liées à la production du maïs et peut renvoyer comme beaucoup de programme à certaines formes d'assistencialisme, il représente une source de revenus toujours bienvenue dans des zones souvent de très grande marginalité socio-économique.

Outre les lourdeurs administratives, un des problèmes majeurs relevés par les paysans renvoie à l'accès trop tardif des subventions. Un paysan du groupe de Comuneros de Villa de Mitla dans les Vallées Centrales de l'État de Oaxaca commente ainsi :

«Ils nous ont bien donné un petit appui qui, comme je vous le disais, n'est pas suffisant pour pouvoir travailler le champ parce que, ce que les gens veulent, c'est que l'appui arrive avant et non après! (...) Les appuis arrivent seulement en septembre alors qu'on a largement passé l'époque des semailles » (Rogelio Sosa, entretien personnel, 28/10/2011).

Le PROMAC ne semble en effet pas échapper aux problèmes de conflits de temporalités liés à de nombreuses politiques environnementales en général (Froger et al. 2009) et plus particulièrement aux programmes de soutien au monde rural mexicain. Il existe un décalage systématique entre les rythmes administratifs et budgétaires et les rythmes agricoles de l'autre. Pour le PROMAC, les convocations et lignes directives du programme sortent généralement au printemps (entre fin février et mai selon les années), à peine quelques temps avant que les principaux travaux des champs qui requièrent le plus d'investissement humain et financiers ne débutent. Les paysans perçoivent le premier des deux versements en automne, à la fin du cycle du maïs dans la plupart des zones, il est donc fort probable que l'argent versé ne serve pas directement à la culture du maïs.

Si le PROMAC n'échappe donc pas totalement à l'assistentialisme et à la bureaucratie de beaucoup de programmes publics mexicains, on peut noter que sa dimension écologique semble rencontrer un écho souvent positif. À propos des ateliers de formations à différentes techniques agroécologiques (conservation des sols, lombricompostage, sélections massales,...) notamment, ceux des paysans qui y ont eu accès soulignent le fait qu'ils y apprennent beaucoup de choses. De la même manière, les foires comme espace de rencontres conviviaux entre paysans, la société civile organisée, les institutions scientifiques et gouvernementales autour du maïs semblent être largement appréciées. Bien qu'il soit difficile d'en mesurer concrètement les effets, il est également possible que ces espaces festifs servent d'une certaine manière de lieu de revalorisation de la culture du maïs auprès des paysans. Il se peut donc bien que, malgré sa portée limitée en termes de conservation, le PROMAC contribue à la diffusion de représentations, savoirs et de pratiques agro-écologiques au sein des communautés dans lequel il intervient, avec toutes les ambiguïtés que cela suppose dans les rapports de force entre savoirs scientifiques et savoirs paysans (Jankowski 2014).

\section{Conclusion}

On a montré comment différents types de savoirs scientifiques, mais aussi gestionnaires étaient en concurrence au stade du projet des grandes lignes du PROMAC. Si différents acteurs et arguments scientifiques sont bien mobilisés pour essayer d'appréhender et de gérer l'objet "agro-biodiversité des maïs mexicains ", on réalise que ce sont plutôt des savoirs-pratiques d'ordre gestionnaire, des habitus institutionnels et des contraintes d'ordre administratif qui conditionnent la mise en 
œuvre du PROMAC. Les communautés épistémiques et leurs savoirs ont dans ce jeu politico-institutionnel bien plus de fonctions (néanmoins essentielles) d'identification du problème, de transcodage (d'une situation socio-environnementale donnée vers la sphère politique) et de légitimation (Roger 2010) que d'orientation pour la mise en place d'une politique rationnelle et efficace, comme dans le modèle de la science based policy. Avec le PROMAC, comme avec de nombreux programmes publics, il serait illusoire de penser que l'action publique traduit mécaniquement des avis scientifiques en une politique rationnelle et surtout efficace. Les effets de traduction ou transcodages aux différentes échelles de la pyramide administrative mexicaine induisent, comme dans une sorte de "téléphone arabe", des effets de distorsion considérables par rapport à un modèle idéal de gouvernance où rationalités scientifiques et politiques s'articuleraient de manière fluide. À chaque échelon, on doit composer avec des compréhensions distinctes, des intérêts, des cultures administratives particulières, des habitudes, des marges de manœuvre, des contraintes et l'on fait bien souvent plus comme on peut, avec les moyens et le temps dont on dispose, qu'appliquer politiquement des savoirs qui redescendraient des sphères scientifiques. Dans cette perspective, il ne reste plus grand chose des savoirs sur la conservation des maïs et, avec eux, des objectifs initiaux du programme. Il ne s'agit cependant pas de faire un bilan purement critique d'un programme qui cherche à élargir les débats sur la biodiversité cultivée aux acteurs directement intéressés (les paysans) pour mener une réflexion sur l'agriculture paysanne et ses nécessités. Il ne s'agit pas non plus de faire un procès en manque de rationalité de l'action publique mexicaine mais bien de montrer au contraire que ces effets de "dissolution" des savoirs scientifiques sont probablement inhérents aux politiques publiques, en général, et, qu'au moins dans le domaine de la biodiversité, la science based policy n'est qu'un mythe bien éloigné des réalités de l'action public et du terrain. En cela, notre étude ne fait que s'inscrire dans la continuité d'autres études de sociologie politique qui déconstruisent la vision d'un État rationnel et efficace (Muller 2000).

À la lumière de sa trajectoire, deux constats émergent. Premièrement, le PROMAC opère au sein de zones caractérisées par une agriculture paysanne, certes marginalisée, mais encore basée sur le riche panel des variétés natives dont leur usage est indépendant des programmes de conservations in situ gouvernementaux ou internationaux. Deuxièmement, le PROMAC n'a donc pas un impact direct sur la conservation des maïs mais, sous la forme d'une subvention environnementale, participe à la construction d'espaces d'expertise, de socialisation et de «bonnes pratiques » autour de la question du maïs natif. Bien que le PROMAC n'atteigne pas l'objectif premier qu'il s'était fixé, il n'est pas dépourvu de fonctions importantes pour les différents acteurs. Pour les paysans, il peut représenter une opportunité d'accès à des ressources financières et cognitives dans le cadre de l'écologisation de leurs pratiques, mais surtout une valorisation symbolique d'un travail - celui du paysan cultivant du maïs natif - historiquement marqué par une marginalisation extrême. Pour les scientifiques qui y participent, il représente une opportunité de rendre visible et mettre en œuvre des approches de conservation innovatrices, bien qu'encore marginales. Pour la CONANP, il représente l'opportunité d'intéresser les paysans à ses missions de conservation. Pour le ministère de l'environnement, il permet d'afficher un respect des cadres institutionnels et légaux en matière de biosécurité. Au niveau des politiques mexicaines agricoles et commerciales, il permet plus stratégiquement de pouvoir afficher un contrepoids environnemental, certes très inégal, mais néanmoins 
mobilisable dans des stratégies de légitimation, aux politiques productivistes et à une éventuelle arrivée des maïs transgéniques.

Si l'expertise scientifique importe, en ce qui concerne la gouvernabilité de la biodiversité et de l'agrobiodiversité, c'est donc bien plus, en rapport avec une dynamique de transcodage, dans la mesure où elle contribue à rendre le problème identifiable, insérable dans l'appareil étatique et traitable, que dans leur capacité à proposer des solutions opérantes. C'est en ce sens que l'expertise scientifique dans le PROMAC servirait en quelque sorte à rendre gouvernable le problème de la conservation des maïs, mais sans le gouverner (Müller 2012).

\section{BIBLIOGRAPHIE}

Altieri M.A. \& Merrick L. 1987 - In situ conservation of crop genetic resources through maintenance of traditional farming systems. Economic Botany 41 (1) : 86-96.

Alvarez-Buylla E. \& Pineyro Nelson A. (Ed.) 2013 - El maíz en peligro ante los transgénicos : un análisis integral sobre el caso de México. México : UNAM, Centro de Investigaciones Interdisciplinarias en Ciencias y Humanidades, Unión de Científicos Comprometidos con la Sociedad.

Akrich M., Callon M. \& Latour B. (Ed.) 2006 - Sociologie de la traduction : textes fondateurs. Paris, Mines ParisTech, les Presses. (Sciences sociales).

Barrera Bassols N. \& Toledo V.M. 2005 - Ethnoecology of the Yucatec Maya: symbolism, knowledge and management of natural resources. Journal of Latin American geography 4 (1).

Bellon M.R. 2004 - Conceptualizing interventions to support on-farm genetic resource conservation. World Development 32 (1) : 159-172.

Boege E. 2008 - El patrimonio biocultural de los pueblos indígenas de México. México, INAH. $345 \mathrm{p}$.

Bonneuil C. \& Joly P.B. 2013 - Sciences, techniques et sociétés. Paris, la Découverte.

Brush S.B. (Ed.) 2000 - Genes in the field: on-farm conservation of crop diversity. Rome, IDRC, Lewis Publisher, IPGRI.

Callon M. 1986 - Éléments pour une sociologie de la traduction. La domestication des coquilles Saint-Jacques dans la Baie de Saint-Brieuc. L'Année sociologique 36 : 169-208.

Chauvet M. 2015 - Biotecnologia y sociedad. México, UAM.

CONABIO 2011 - Proyecto global "Recopilación, generación, actualización y análisis de información acerca de la diversidad genética de maíces y sus parientes silvestres en México". México, CONABIO. En ligne : https://www.biodiversidad.gob.mx/media/1/genes/files/InformedeGestion_V1.pdf

CONABIO 2020 - Razas de maíz de México. México, CONABIO. En ligne : https:// www.biodiversidad.gob.mx/diversidad/alimentos/maices/razas-de-maiz CONEVAL 2012 - Programa de Conservación del Maíz Criollo (PROMAC). Evaluación de consistencia y resultados 2011-2012, resumen ejecutivo. 
Dahan A. \& Guillemot H. 2015 - Les relations entre science et politique dans le régime climatique : à la recherche d'un nouveau modèle d'expertise ? Natures, Sciences, Sociétés $23: 6-18$.

De Boef W.S., Subedi A., Peroni N., Thijssen M. \& O'Keeffe E. (Ed.). 2013 - Community Biodiversity Management: Promoting resilience and the conservation of plant genetic resources. Routledge.

Dumoulin D. 2003 - Les politiques de la nature confrontées aux politiques du renouveau indien: une étude transnationale depuis le Mexique. Thèse de doctorat de l'Institut d'Études Politiques de Paris (Dir. Hermet), $937 \mathrm{p}$.

Foyer J. 2015 - Défendre les maïs au Mexique, entre ressources génétiques et ressources politiques. In : Thomas F. \& Boisvert V. (Ed.) Le pouvoir de la biodiversité. Paris, IRD Éditions/Quae. (Objectifs Suds).

Foyer J. \& Bonneuil C. 2015 - La bioseguridad mexicana : una « actuación de seriedad ». Revista Mexicana de Sociologia 77 (1) : 37-68.

Foyer J \& Dumoulin Kervran D. 2015 - Environmentalism of NGOs versus Environmentalism of the Poor? Mexico's Social Environmentalism Coalitions. In : Almeida P. \& Cordero Ulate A. (Ed.) Handbook of Social Movements across Latin America. Springer : 223-235.

Froger G., Geronimi V., Meral P \& Schembri P. (Ed.) 2009 - Diversité des politiques de développement durable. Temporalités et durabilités en conflit à Madagascar, au Mali et au Mexique. Paris, Gemdev et Karthala.

Garibay R. 2010 - Analisis del programa de conservación de maíz criollo y propuesta para el 2011. Document administratif de la CONANP.

Garibay R. 2012 - Programa de Conservación del Maíz Criollo, la Milpa y la Agrobiodiversidad de la CONANP. Communication personnelle, manuscrit non publié.

Gérard E. 2014 - La construction politique et sociologique des savoirs sur le maïs au prisme du "Projet global sur les maïs primitifs" (Mexique, 2006-2010). Engov Field Reports

Haas M.P. 1989 - Do regime matter? Epistemic communities and Mediterranean pollution control. International Organization 43 (3) : 377-403.

Hackett E.J., Amsterdamska O., Lynch M. \& Wajcman J. (Ed.) 2008 - The handbook of science and technology studies. 3rd ed. Cambridge, USA The MIT Press.

Instituto para la gestión integral de cuencas hidrográficas A.C. 2009 - Evaluación en materia de diseño del programa de conservación del maíz criollo, informe final.

Hope R. 1999 - Policy analysis, science and politics: from 'speaking truth to power' to 'making sense together'. Science and Public Policy 26 (3) : 201-210.

Jankowski F. 2014 - La diffusion de savoirs agro-écologiques dans l'État de Oaxaca (Mexique). Revue d'anthropologie des connaissances 8 (3) : 619-641.

Jarvis D.I., Hodgkin T., Sthapit B.R., Fadda C. \& Lopez-Noriega I. 2011 - An heuristic framework for identifying multiple ways of supporting the conservation and use of traditional crop varieties within the agricultural production system. Critical Reviews in Plant Sciences, 30 (1-2) : 125-176.

Jasanoff S. \& Martello M.L. 2004 - Earthly Politics: local and global in enviropnemental governance. Cambridge, MIT Press.

Kato T.A., Mapes C., Mera L.M., Serratos J.A. \& Bye R.A. 2009 - Origen y diversificación del maíz: una revisión analítica. México, D.F., Universidad Nacional Autónoma deMéxico, Comisión Nacional para el Conocimiento y Uso de la Biodiversidad. 
King D. \& Le Galès, P. 2011 - Sociologie de l'État en recomposition. Revue Française de Sociologie $523: 453-480$.

Lascoumes P. 1994 - L'éco-pouvoir : environnements et politique. Paris, La découverte, 324 p.

Lascoumes P. 1996 - Rendre gouvernable : de la «traduction » au «transcodage » : l'analyse des processus de changement dans les réseaux d'action publique. La Gouvernabilité, CURAPP, Paris, Presses universitaires de France : 325-338.

Lascoumes P. \& Le Galès P. 2012 - Sociologie de l'action publique. Paris, Armand Colin.

Lazos Chavero E. \& Chauvet M. 2011 - Análisis del contexto social y biocultural de las colectas de maíces nativos en México. México, CONABIO.

Léonard E. \& Foyer J. 2011 - De la integración nacional al desarrollo sustentable. Trayectoria nacional y producción local de la política rural en México. México, CEDRSSA, IRD, 466 p.

Louette D., Charrier A. \& Berthaud J. 1997 - In situ conservation of maize in Mexico: genetic diversity and maize seed management in a traditional community. Economic botany 51 (1) : 20-38.

Marielle C \& Peralta L. 2007 - La contaminacion transgénica del maiz en México. Luchas civiles en defensa del maiz y la soberania alimentaria. México, GEA.

Muller P. 2000 - L'analyse cognitive des politiques publiques : vers une sociologie politique de l'action publique. Revue française de science politique 50 (2) : 189-208.

Müller B. 2012 - Comment rendre le monde gouvernable sans le gouverner: les organisations internationales analysées par les anthropologues. Critique internationale (1) : 9-18.

Nations J.D. \& Nigh R. 1980 - The evolutionary potential of Lacandon Maya sustained-yield tropical forest agriculture. J Anthropol Res $36: 1-30$.

Padioleau J.G. 1982 - L'État au concret. Presses universitaires de France.

Perales H. \& Golicher D. 2011 - Modelos de Distribución para las Razas de Maíz en México y Propuesta de Centros de Diversidad y de Provincias Bioculturales. México, Conabio.

Perales H. \& Golicher D. 2014 - Mapping the diversity of maize races in Mexico. PloS one 9 (12) : e114657.

Perales H. 2017 - Informe Final del Proyecto NM001 Propuesta para formular un marco conceptual para las actividades de conservación de las variedades nativas de maíz. CONABIO.

Pestre D. 2006 - Introduction aux Science Studies. Paris, La Découverte, 122 p. (Repères).

Roger A. 2010 - Constructions savantes et légitimation des politiques européennes : la circulation des savoirs sur la vigne et le vin. Revue Française de Science Politique 6 (60) : 1091-1113.

Sánchez G.J.J. 2011 - Diversidad del Maíz y el Teocintle. Informe preparado para el proyecto: "Recopilación, generación, actualización y análisis de información acerca de la diversidad genética de maíces y sus parientes silvestres en México". Comisión Nacional para el Conocimiento y Uso de la Biodiversidad. Manuscrito.

Scott J.C. 1998 - Seeing Like a State: How Certain Schemes to Improve the Human Condition Have Failed. New Haven, CT: Yale University Press.

Toledo V.M. 2000 - La Paz en Chiapas. Ecología, luchas indígenas y modernidad alternativa. México, Quinto Sol, 253 p.

Weaver K. 1986 - The politics of blame avoidance. Journal of Public Policy 6 (4) : 371-398. 


\section{ANNEXES}

Principaux sigles utilisés :

CONANP : Comisión Nacional de Areas Naturales Protegidas

CONABIO: Comisión Nacional para el conocimiento y uso de la Biodiversidad

CONEVAL : Comision Nacional de Evaluacion de la Politica de Desarrollo Social

PROMAC : Programa de Maíces Criollos

PROMAF : Programa de Maíz y Frijol

PROCAMPO : Programa de Apoyos Directos al Campo

PGMN : Programa Global de Maices Nativos

\section{NOTES}

1. Pour Jean Foyer, cette recherche a été menée dans le cadre du projet Bekonal Building and Exchanging "Knowledges" on Natural Resources (coordination Mina Kleich, IRD) du consortium Européen ENGOV Environmental Governance in Latina America and the Caribbean entre 2011 et 2015. Pour Mariana Fenzi, elle a été menée dans le cadre d'une recherche doctorale soutenue par le Centre Alexandre Koyré (EHESS/CNRS/MNHN).

2. En 2019, sous la présidence de Andrés Manuel López Obrador, la SAGARPA change de nom et devient Secretaría de Agricultura y Desarrollo Rural (SADER).

3. Les actions du PROMAC ont commencé en 2009 et le programme a été clôturé en 2015. Les détails par année sont consultables sur: https://www.gob.mx/conanp/acciones-y-programas/ maiz-criollo

4. https://www.gob.mx/conanp/acciones-y-programas/maiz-criollo.

5. Nous avons également pu collecter sur le site internet de la CONANP et auprès de nos interlocuteurs, un important corpus de différents documents administratifs (comptes rendus de réunions, rapports d'évaluation, formulaires...).

6. Criollo.

7. L'information générée par ce projet est disponible sur le site internet de la CONABIO : https:// www.biodiversidad.gob.mx/diversidad/proyectoMaices

8. L'Instituto Nacional de Ecología y Cambio Climático (INECC) a un rôle également plus technique de production de données et de formation de personnel. La Procuradoria Federal de Proteccion al Ambiente (PROFEPA) a quant à elle plus un rôle de surveillance des différentes zones protégées.

9. Notamment le PROCODES (anciennement PRODERS), un des programmes emblématiques du développement durable intégral promu par la SEMARNAT dès sa création au milieu des années 90 (Dumoulin 2003, Léonard \& Foyer 2011). Avec un budget d'environ 210 millions de pesos en 2012, ce programme visant à subventionner des activités productives dans les Aires Naturelles Protégées, était le plus important de ce type au sein de la CONANP.

10. La red de Etnoecología y del Patrimonio Biocultural de México (REPB) a comme principal objectif «...la connaissance, la revalorisation et la défense du Patrimoine Bioculturel du Mexique». Ce patrimoine se reconnaît par la jonction de la diversité biologique, de la diversité culturelle ou linguistique et l'agro-diversité, traslape qui a lieu dans des territoires bien définis du pays et dont les principaux acteurs mais non les seuls, sont les villages indigènes et leurs communautés. http://etnoecologia.uv.mx/Red_Introduccion_iframe.html

11. Cette dimension est d'autant plus importante dans le contexte mexicain qu'une majeure partie des zones protégées sont la propriété des communautés indigènes (biens communaux) ou des ejidos paysans. 
12. Pour donner un ordre d'idée, l'appui du PROMAC oscille entre 1200 pesos et 1700 pesos à l'hectare selon les années; le programme n'appuie généralement pas plus d'un ha par personne. Dans différentes communautés de Oaxaca et Puebla où l'on a pu mener les enquêtes, on estimait que l'investissement nécessaire à l'hectare oscillait entre 2500 et 3000 pesos, essentiellement en main d'œuvre pour différentes tâches (préparation du terrain, semailles, nettoyage des parcelles, désherbage,...) et pour la location d'un attelage cheval/charrue ou d'un tracteur.

\section{RÉSUMÉS}

Cet article propose une analyse du programme phare de conservation de la diversité des maïs mexicains - le Programa de Conservación de Maíz Criollo (PROMAC)- depuis son dessein au niveau des institutions nationales jusqu'à sa mise en œuvre au niveau local, en passant par l'échelon administratif régional. Nous montrons comment les différents types de savoirs mobilisés contribuent ou non aux diverses opérations pour rendre le problème de la conservation du maïs lisible et gouvernable. Nous soulignons également comment d'autres types de facteurs (dépendance au sentier, habitus institutionnels et administratifs, manque de ressources) s'avèrent être des forces déterminantes dans la réalisation du programme.

This article offers an analysis of the flagship program for the conservation of the diversity of Mexican maize - the Programa de Conservación de Maíz Criollo (PROMAC) - from its design at the level of national institutions to its implementation at the local level, including the regional administrative level. We show whether or not the different types of knowledge mobilized contribute to the various operations meant to make the issue of maize landraces conservation readable and governable. We also highlight how other types of factors (path dependence, institutional and administrative habits, lack of resources) turn out to be determining forces in the realization of the program.

Este artículo propone un análisis del programa modelo de conservación de la diversidad de los maíces mexicanos -Programa de Conservación de Maíz Criollo (PROMAC)- desde su diseño a nivel de las instituciones nacionales hasta su implementación a nivel local, incluyendo la escala administrativa regional. Demostramos cómo los diferentes tipos de saberes movilizados contribuyen o no a las múltiples operaciones dirigidas a volver gobernable y visible el problema de la conservación del maíz. Al mismo tiempo, señalamos de qué forma otros tipos de factores (dependencia del camino, habitus institucionales y administrativos, falta de recursos, etc.) resultan ser elementos decisivos en la implementación del programa.

\section{INDEX}

Index géographique : Mexique

Keywords : agrobiodiversity, conservation, maize, Mexico, public policy, science

Palabras claves : agrobiodiversidad, conservación, maíz, México, políticas públicas, ciencias

Mots-clés : agrobiodiversité, conservation, maïs, politiques publiques, sciences 


\section{AUTEURS}

\section{JEAN FOYER}

Centre de Recherche et de Documentation des Amériques/CNRS, Paris, France

foyerjean@gmail.com

\section{MARIANNA FENZI}

Centre Alexandre Koyré, EHESS - Laboratory for the History of Science and Technology, EPFL, Lausanne, Suisse.marianna.fenzi@epfl.ch 